\title{
Confiabilidade dos diagnósticos de internações por causas externas financiadas pelo Sistema Único de Saúde em dois municípios do Estado do Paraná, Brasil
}

\author{
Reliability of the diagnoses of hospital admissions \\ for external causes financed by the Brazilian Unified Health \\ System-SUS in two cities in the State of Paraná, Brazil
}

\author{
Thais Aidar de Freitas Mathias ${ }^{1}$ \\ Selma Maffei de Andrade ${ }^{2}$ \\ Maria de Fátima Akemi Iwakura Tomimatsu ${ }^{3}$ \\ Dorotéia Fátima Pelissari de Paula Soares ${ }^{1}$ \\ Maria da Penha Marques Sapata ${ }^{4}$ \\ Andressa Suellen Frascarelli ${ }^{5}$ \\ Regina Kazue Tanno de Souza ${ }^{2}$
}

${ }^{1}$ Departamento de Enfermagem, Centro de Ciências da Saúde, Universidade Estadual de Maringá. Av. Colombo 5790, Zona 7. 87.020900 Maringá PR Brasil. tafmathias@gmail.com ${ }^{2}$ Departamento de Saúde Coletiva, Universidade Estadual de Londrina.

${ }^{3}$ Autarquia Municipal de Saúde de Londrina.

${ }^{4}$ Secretaria Municipal de Saúde de Maringá.

${ }^{5}$ Enfermeira graduada pela Universidade Estadual de Maringá.

\begin{abstract}
The scope of this article is to analyze the reliability of the diagnoses of hospital admissions for external causes financed by the Brazilian Unified Health System (SUS). The diagnoses for hospital admissions from the Hospital Information System (SIH-SUS) data were compared with those from the research panel created from information culled from authorizations for hospital admission (AIH) complemented with information from other sources. The reliability was analyzed in groups using crude agreement and Kappa statistics. The crude agreement was $41.1 \%$ in Londrina and $15.8 \%$ in Maringá and the highest reliability was found for groupings for self-inflicted lesions and traffic accidents in Londrina (Kappa 0.57 and 0.56) while in Maringá reliability was acceptable for self-inflicted lesions (Kappa 0.28). The low agreement with differentiated standards according to groupings of causes and according to the municipalities highlight the fact that efforts must be made to improve the quality and use of the SIH-SUS in order to understand and intervene in accidents and violence in Brazil.

Key words Hospital admissions, Hospital information system, External causes, Reliability, Reproducibility of results
\end{abstract}

Resumo $O$ objetivo deste artigo é analisar a confiabilidade dos diagnósticos de internações por causas externas do Sistema Único de Saúde (SUS). Os diagnósticos de internação do Sistema de Informações Hospitalares-SUS (SIH-SUS) foram comparados aos do banco pesquisa, construído com informações das autorizações de internação hospitalar (AIH) complementadas por outras fontes. A confiabilidade foi analisada por agrupamentos utilizando concordância bruta e estatística Kappa. A concordância bruta foi de 41,1\% em Londrina e 15,8\% em Maringá e a melhor confiabilidade foi a moderada para os agrupamentos das lesões autoprovocadas e acidentes de transporte em Londrina (kappa 0,57 e 0,56), ao passo que em Maringá foi a aceitável para as lesões autoprovocadas (kappa 0,28). As baixas concordâncias com padrões diferenciados segundo os agrupamentos de causas e segundo os municípios evidenciam que esforços devem ser empreendidos para melhorar a qualidade e utilização do SIHSUS para conhecer e intervir nos acidentes e violências no Brasil.

Palavras-chave Internações hospitalares, Sistema de informação hospitalar, Causas externas, Confiabilidade, Reprodutibilidade de resultados 


\section{Introdução}

Os acidentes e as violências representam um dos principais desafios da atualidade para o setor da saúde, da segurança pública e para toda a sociedade, pois são responsáveis por mais de 5 milhões de mortes e milhares de internações em todo mundo ${ }^{1}$. No Brasil, segundo dados do Datasus, as lesões traumáticas e os ferimentos fatais e não fatais, consequências de causas externas, vêm aumentando e os dados revelam que as mortes por causas externas passaram de 59 por 100 mil habitantes em 1980 para 74,3 em 2010².

No Brasil, o monitoramento das violências para fins de vigilância epidemiológica é realizado por meio de dados da declaração de óbito e da autorização de internação em hospitais públicos, disponíveis no Sistema de Informações sobre Mortalidade (SIM) e Sistema de Informações Hospitalares do Sistema Único de Saúde (SIHSUS), constituindo importante ferramenta para o conhecimento da carga das violências no país ${ }^{3}$. Ainda, para complementar os dados já disponíveis no SIM e SIH-SUS, o Ministério da Saúde implantou, em 2006, o Sistema de Vigilância de Violências e Acidentes (Viva). A proposta do Viva é realizar inquéritos periódicos em municípios previamente selecionados para monitoramento das violências e acidentes em serviços de saúde sentinela e para a vigilância contínua das violências doméstica, sexual e outras ${ }^{3,4}$.

Considerando que as ocorrências de causas externas são em sua maioria não fatais, é importante que esses eventos sejam analisados para a adequação de estratégias preventivas de enfrentamento do problema. Para isso, é imprescindível que os sistemas de informações em saúde, de mortalidade e morbidade por causas externas sejam de boa qualidade e constantemente aperfeiçoados para a prevenção do problema e atenção integral às vítimas ${ }^{3}$. Tendo em vista que as estatísticas de internações hospitalares têm sido cada vez mais utilizadas para estudos de morbidade por causas externas, faz-se necessário que essas fontes de dados sejam monitoradas para garantir informações completas e confiáveis, fato que justifica estudos que analisem tanto o perfil epidemiológico das internações como a qualidade dos dados disponíveis.

Estima-se que as internações hospitalares financiadas pelo setor público no Brasil representem cerca de $75 \%$ das internações hospitalares, com variações entre as regiões e os estados brasileiros, em função da população que utiliza planos de saúde privados, da existência de serviços hos- pitalares disponíveis e do acesso da população a esses serviços ${ }^{5}$. Alguns dados desse contingente de internações estão disponíveis no banco de dados do Sistema de Informações Hospitalares do Sistema Único de Saúde (SIH-SUS), o maior sistema de informação em saúde nacional, que registrou, em 2011, cerca de 11 milhões de internações em hospitais próprios e/ou contratados. Seu objetivo principal está voltado para o pagamento das internações realizadas tanto em hospitais públicos quanto nos privados ou filantrópicos conveniados ao SUS. As informações que alimentam o SIH-SUS são provenientes da Autorização de Internação Hospitalar (AIH) que é o documento que compõe cada registro de sua base de dados.

Desde janeiro de 1998, tornou-se obrigatório o preenchimento, além da informação sobre o diagnóstico principal ou lesão que motivou a internação, também a relativa ao diagnóstico secundário, que consiste na causa externa (acidentes, violências ou agressões). No diagnóstico principal, o código a ser preenchido corresponde ao capítulo XIX - Lesões, envenenamentos e algumas outras consequências de causas externas (S00 a T98) da Classificação Internacional de Doenças - 10a Revisão, enquanto que o diagnóstico secundário remete-se ao capítulo XX - Causas Externas de Morbidade e Mortalidade (V01 a Y98).

A análise epidemiológica das hospitalizações por causas externas representa um grande desafio à saúde pública. Para sua investigação são encontradas dificuldades sob diversos aspectos, tais como: sistemas de informações hospitalares com maior ênfase no faturamento, a falta de integração destes com os sistemas de saúde público e privado, assim como o preenchimento inadequado dos prontuários ${ }^{6}$ e documentos médicos para o preenchimento da AIH e codificação dos diagnósticos de internação de acordo com a CID-10.

Desta forma, destaca-se a possibilidade de subnotificação dos diagnósticos secundários, ou das causas externas de internação quando analisadas tendo como fonte o SIH-SUS, que podem estar classificadas como eventos de intenção indeterminada, cuja causa é desconhecida, ou ainda como causa não externa ${ }^{7}$, ou causa externa diversa da verdadeira, dificultando o conhecimento do real perfil de internações. Em pesquisa realizada anteriormente em Londrina e Maringá, Paraná ${ }^{7}$, foram avaliadas a especificidade e a sensibilidade do SIH, mas para o conjunto das causas externas e sem observar a concordância dos diagnósticos por pares e sem consulta aos prontuários, fonte que foi acrescentada no presente estudo. Assim, o 
Este estudo faz parte e amplia a análise de trabalho anterior ${ }^{7}$. Neste, buscou-se analisar a confiabilidade da informação registrada no SIH-SUS, acrescentando à investigação dados das internações por causas externas em prontuários hospitalares visando à redução da proporção de causas externas de intenção indeterminada já descrita naquele estudo. Naquele ${ }^{7}$, aventou-se a possibilidade de sub-registro de algumas causas, especialmente das internações por acidentes por exposição a forças mecânicas inanimadas, agressões e lesões autoprovocadas, porém, como já mencionado, a análise não foi feita por pares de registros entre as diferentes fontes de informação.

O banco de dados da pesquisa foi composto por todas as internações por causas externas financiadas pelo SUS ocorridas nos municípios de Londrina e Maringá no ano de 2004. As internações foram identificadas nos setores de Auditoria das Secretarias de Saúde dos municípios por meio de levantamento manual de todos os laudos médicos de cada internação. Foram selecionados todos os laudos médicos com registro de alguma lesão, envenenamento ou causa externa como diagnóstico ou como motivo da internação. Para os laudos médicos que tinham diagnósticos de lesão sem menção de causa externa ou mencionando evento de intenção indeterminada, outras fontes foram consultadas, com o objetivo de qualificar ao máximo o banco de dados.

A primeira fonte consultada foi o Sistema de Informação sobre Mortalidade (SIM), seguida pela busca em registros do Sistema de Atendimento ao Trauma e às Emergências (SIATE) dos dois municípios, conforme já descrito em estudo anterior ${ }^{7}$. Para o presente estudo, o banco de dados foi complementado voltando-se a cada hospital onde ocorreu a internação, identificando e consultando cada prontuário, para reduzir os diagnósticos de causas externas por intenção indeterminada.

As informações que compuseram o banco de dados da pesquisa foram coletadas e transcritas em fichas e os diagnósticos foram codificados por profissionais treinados segundo a CID-10, com discussão de divergências na codificação até consenso. Foi realizada dupla digitação dos dados para verificação e correção de possíveis erros, por meio de programa Epi Info versão 6.04. Essas internações formaram o banco de dados considerado padrão ouro, já que foram coletadas informações diretamente do laudo médico, que é parte integrante do prontuário do paciente, com posterior complementação dos dados conforme relatado. Paralelamente, outro banco de dados foi composto selecionando-se todas as internações diretamente do SIH-SUS ocorridas nos dois municípios, referentes ao mesmo período.

Os dois bancos de dados foram ligados por meio do número da AIH para proceder a comparação dos diagnósticos em ambas as fontes de dados (pesquisa e SIH-SUS) em pares de registros, que foram analisados por agrupamentos da CID-10: Acidente de transporte (V01-V99), Quedas (W00-W19), Exposição a forças mecânicas inanimadas (W20-W49), Lesões autoprovocadas (X60-X84), Demais acidentes (W50-W59; Y40-Y84), Agressões (X85-Y09; Y35-Y36), Intenção indeterminada (Y10-Y34), Sequelas de causas externas de morbidade e mortalidade (Y85-Y89) e causas não externas. Para análise da concordância foram comparados os códigos das causas do banco pesquisa com os códigos do banco do SIHSUS utilizando a estatística Kappa ${ }^{8}$, calculada no programa WinPepi ${ }^{9}$ e usando a classificação proposta por Landis e Kock ${ }^{10}$, que apresentam as seguintes categorias conforme a força da concordância: 0,81 a 1,00 (quase perfeita), 0,61 a 0,80 (substancial), 0,41 a 0,60 (moderada), 0,21 a 0,40 (aceitável), 0,00 a 0,20 (fraca) e $<0,00$ (pobre). O projeto de pesquisa foi aprovado pelos Comitês de Ética das Universidades Estaduais de Londrina e de Maringá.

\section{Resultados}

Após a construção do banco de dados da pesquisa observou-se que do total de 4018 internações por causas externas de Londrina e $2370 \mathrm{de} \mathrm{Ma-}$ ringá coletadas dos laudos médicos, as ocorridas por intenção indeterminada representaram inicialmente, respectivamente $32,3 \%$ e $40,6 \%$. Após a consulta às diferentes fontes de dados (SIM, SIATE e prontuários médicos) essas causas foram reduzidas para 10,3\% para Londrina e 25\% para Maringá. A fonte que mais contribuiu e acrescentou dados sobre causas externas específicas ao banco pesquisa foi o prontuário do paciente, para os dois municípios (Tabela 1). No SIH-SUS os percentuais de causas externas de intenção in- 
sões, lesões autoprovocadas e acidentes de transporte (kappa 0,57; 0,56 e 0,45, respectivamente). Em Maringá, a concordância entre os bancos de dados foi inferior à de Londrina para todos os agrupamentos, pois apenas para as lesões autoprovocadas foi observada concordância considerada aceitável (kappa 0,28) (Tabela 2).

As Tabelas 3 e 4 mostram a matriz de distribuição das internações por causas externas segundo agrupamentos, nos dois bancos de dados e respectivos percentuais de concordância bruta, que em Londrina foram maiores para todos os agrupamentos. O percentual de concordância mais elevado foi observado para as quedas nos dois municípios (72,5\% para Londrina e 31,2\% para Maringá) e os mais baixos para exposição a forças mecânicas inanimadas em Londrina $(24,3 \%)$ e agressões em Maringá (1,9\%). Das 1136 internações por acidentes de transporte em Londrina, apenas 440 concordaram com os dados do SIH-SUS. Entre as discordantes 423 foram registradas no SIH-SUS como quedas e 221 não

Tabela 2. Concordância segundo agrupamentos de causas externas. Londrina e Maringá, 2004.

\begin{tabular}{|c|c|c|c|c|c|c|}
\hline \multirow{3}{*}{ Agrupamentos } & \multicolumn{6}{|c|}{ Londrina } \\
\hline & \multicolumn{2}{|c|}{ pesquisa } & \multicolumn{2}{|c|}{ SIH } & \multirow[b]{2}{*}{ kappa } & \multirow[b]{2}{*}{ IC-95\% } \\
\hline & $\mathbf{n}$ & $\%$ & $\mathbf{n}$ & $\%$ & & \\
\hline Acidentes de transporte (V01-V99) & 1.136 & 28,3 & 492 & 16,4 & 0,45 & 0,$42 ; 0,48$ \\
\hline Quedas (W00-W19) & 974 & 24,3 & 1.703 & 56,7 & 0,32 & 0,$29 ; 0,34$ \\
\hline Exp forças inanimadas (W20-W49) & 267 & 6,6 & 106 & 3,5 & 0,32 & 0,$26 ; 0,39$ \\
\hline Demais acidentes (W50-X59; Y40-Y84) & 684 & 17,0 & 385 & 12,8 & 0,39 & 0,$35 ; 0,43$ \\
\hline Lesões autoprovocadas (X60-X84) & 76 & 1,9 & 61 & 2,0 & 0,56 & 0,$46 ; 0,66$ \\
\hline Agressões (X85-Y09;Y35-Y36) & 387 & 9,6 & 195 & 6,5 & 0,57 & 0,$53 ; 0,62$ \\
\hline Intenção indeterminada (Y10-Y34) & 412 & 10,3 & 57 & 1,9 & 0,00 & $-0,02 ; 0,02$ \\
\hline Sequelas (Y85-Y89) & 82 & 2,0 & 3 & 0,1 & $-0,01$ & $-0,01 ; 0,00$ \\
\hline TOTAL & 4.018 & - & 3.002 & - & 0,46 & 0,$43 ; 0,49$ \\
\hline Causa não externa & 70 & - & 1.086 & - & - & - \\
\hline \multirow{3}{*}{ Agrupamentos } & \multicolumn{6}{|c|}{ Maringá } \\
\hline & \multicolumn{2}{|c|}{ pesquisa } & \multicolumn{2}{|c|}{ SIH } & \multirow[b]{2}{*}{ kappa } & \multirow[b]{2}{*}{ IC-95\% } \\
\hline & $\mathbf{n}$ & $\%$ & $\mathbf{n}$ & $\%$ & & \\
\hline Acidentes de transporte (V01-V99) & 567 & 23,9 & 517 & 36,8 & 0,05 & 0,$01 ; 0,10$ \\
\hline Quedas (W00-W19) & 683 & 28,8 & 709 & 50,6 & 0,03 & $-0,02 ; 0,07$ \\
\hline Exp forças inanimadas (W20-W49) & 107 & 4,5 & 14 & 1,0 & 0,07 & 0,$01 ; 0,14$ \\
\hline Demais acidentes (W50-X59; Y40-Y84) & 272 & 11,5 & 38 & 2,7 & 0,08 & 0,$03 ; 0,12$ \\
\hline Lesões autoprovocadas (X60-X84) & 14 & 0,6 & 7 & 0,5 & 0,28 & 0,$03 ; 0,54$ \\
\hline Agressões (X85-Y09;Y35-Y36) & 106 & 4,5 & 4 & 0,3 & 0,03 & $-0,01 ; 0,08$ \\
\hline Intenção indeterminada (Y10-Y34) & 592 & 25,0 & 2 & 0,1 & $-0,01$ & $-0,01 ; 0,00$ \\
\hline Sequelas (Y85-Y89) & 29 & 1,2 & 112 & 8,0 & $-0,02$ & $-0,03 ; 0,01$ \\
\hline TOTAL & 2.370 & - & 1.403 & - & 0,20 & 0,$18 ; 0,23$ \\
\hline Causa não externa & 45 & - & 1.012 & - & - & - \\
\hline
\end{tabular}

*Percentual calculado excluindo as "causas não externas" para cada banco de dados (pesquisa e SIH-SUS) de cada município. 
Tabela 3. Distribuição das internações conforme a concordância da informação do SIH em relação à da pesquisa (padrão-ouro). Londrina, 2004.

\begin{tabular}{|c|c|c|c|c|c|}
\hline \multirow{2}{*}{ SIH-SUS } & \multicolumn{5}{|c|}{ pesquisa } \\
\hline & $\begin{array}{c}\text { Não } \\
\text { externa }\end{array}$ & $\begin{array}{l}\text { Acidentes } \\
\text { transp. }\end{array}$ & Quedas $\quad \begin{array}{c}\text { Ex } \\
\text { ina }\end{array}$ & $\begin{array}{l}\text { xp forças } \\
\text { lanimadas }\end{array}$ & $\begin{array}{c}\text { Demais } \\
\text { acidentes }\end{array}$ \\
\hline Causa não externa & - & 221 & 222 & 79 & 235 \\
\hline Acidentes de transporte (V01-V99) & 4 & 440 & 15 & 4 & 6 \\
\hline Quedas (W00-W19) & 57 & 423 & 706 & 82 & 170 \\
\hline Expos. a forças mecânicas inanimadas (W20-W49) & - & 4 & 5 & 65 & 6 \\
\hline Demais acidentes (W50-X59;Y40-Y84) & 4 & 37 & 22 & 21 & 248 \\
\hline Lesões autoprovocadas (X60-X84) & 2 & - & - & - & 7 \\
\hline Agressões (X85-Y09;Y35-Y36) & - & 1 & 2 & 6 & 3 \\
\hline Intenção indeterminada (Y10-Y34) & 3 & 10 & 2 & 9 & 7 \\
\hline Sequelas (Y85-Y89) & - & - & - & 1 & 2 \\
\hline TOTAL & 70 & 1.136 & 974 & 267 & 684 \\
\hline Concordância bruta $(41,1 \%)$ & - & 38,5 & 72,5 & 24,3 & 36,2 \\
\hline \multirow{2}{*}{ SIH-SUS } & \multicolumn{5}{|c|}{ pesquisa } \\
\hline & $\begin{array}{l}\text { Lesões } \\
\text { autopr. }\end{array}$ & Agressões & $\begin{array}{c}\text { Intenção } \\
\text { indeterminada }\end{array}$ & Sequelas & TOTAL \\
\hline Causa não externa & 13 & 77 & 195 & 44 & 1.086 \\
\hline Acidentes de transporte (V01-V99) & 3 & 6 & 13 & 1 & 492 \\
\hline Quedas (W00-W19) & 5 & 86 & 142 & 32 & 1.703 \\
\hline Expos. a forças mecânicas inanimadas (W20-W49) & - & 16 & 10 & - & 106 \\
\hline Demais acidentes (W50-X59;Y40-Y84) & 7 & 13 & 28 & 5 & 385 \\
\hline Lesões autoprovocadas (X60-X84) & 39 & 2 & 11 & - & 61 \\
\hline Agressões (X85-Y09;Y35-Y36) & 1 & 175 & 7 & - & 195 \\
\hline Intenção indeterminada (Y10-Y34) & 8 & 12 & 6 & - & 57 \\
\hline Sequelas (Y85-Y89) & - & - & - & - & 3 \\
\hline TOTAL & 76 & 387 & 412 & 82 & 4.088 \\
\hline Concordância bruta $(41,1 \%)$ & 51,3 & 45,2 & 1,5 & - & - \\
\hline
\end{tabular}

foram reconhecidas como causas externas (Tabela 3). Da mesma forma em Maringá, pois das 567 internações por acidentes de transporte no banco pesquisa, 206 estavam no SIH-SUS como quedas e 173 também não foram reconhecidas como causas externas (Tabela 4).

Das 387 internações por agressões em Londrina o SIH-SUS registrou 86 como quedas e 77 não foram consideradas causas externas (Tabela 3). Em Maringá, das 106 internações por agressões 62 não foram consideradas causas externas, 25 o foram como acidentes de transporte e 14 como quedas, segundo o banco do SIH-SUS (Tabela 4 ).

\section{Discussão}

Os resultados mostraram primeiramente que o banco de dados do SIH-SUS apresenta algumas limitações quando o objetivo é análise das inter- nações hospitalares por causas externas e ainda que houve variação importante dos dados, entre os dois municípios. Foi observado que ao analisar a confiabilidade do SIH-SUS por agrupamentos de diagnósticos a melhor concordância encontrada foi a "moderada", que ocorreu no município de Londrina para as internações por agressões, lesões autoprovocadas e acidentes de transporte (kappa 0,57; 0,56 e 0,45, respectivamente). Para o município de Maringá a concordância entre os bancos de dados pesquisa e SIH-SUS foi inferior à de Londrina para todos os agrupamentos; apenas para as internações por lesões autoprovocadas foi observada concordância considerada "aceitável” (kappa 0,28).

Embora haja sub-registro de causas externas no banco de dados oficial, foi observado que o SIH-SUS informou menor percentual de internações no agrupamento de intenção indeterminada, fato ocorrido nos dois municípios. Esse resultado sugere que, mesmo não existindo a 


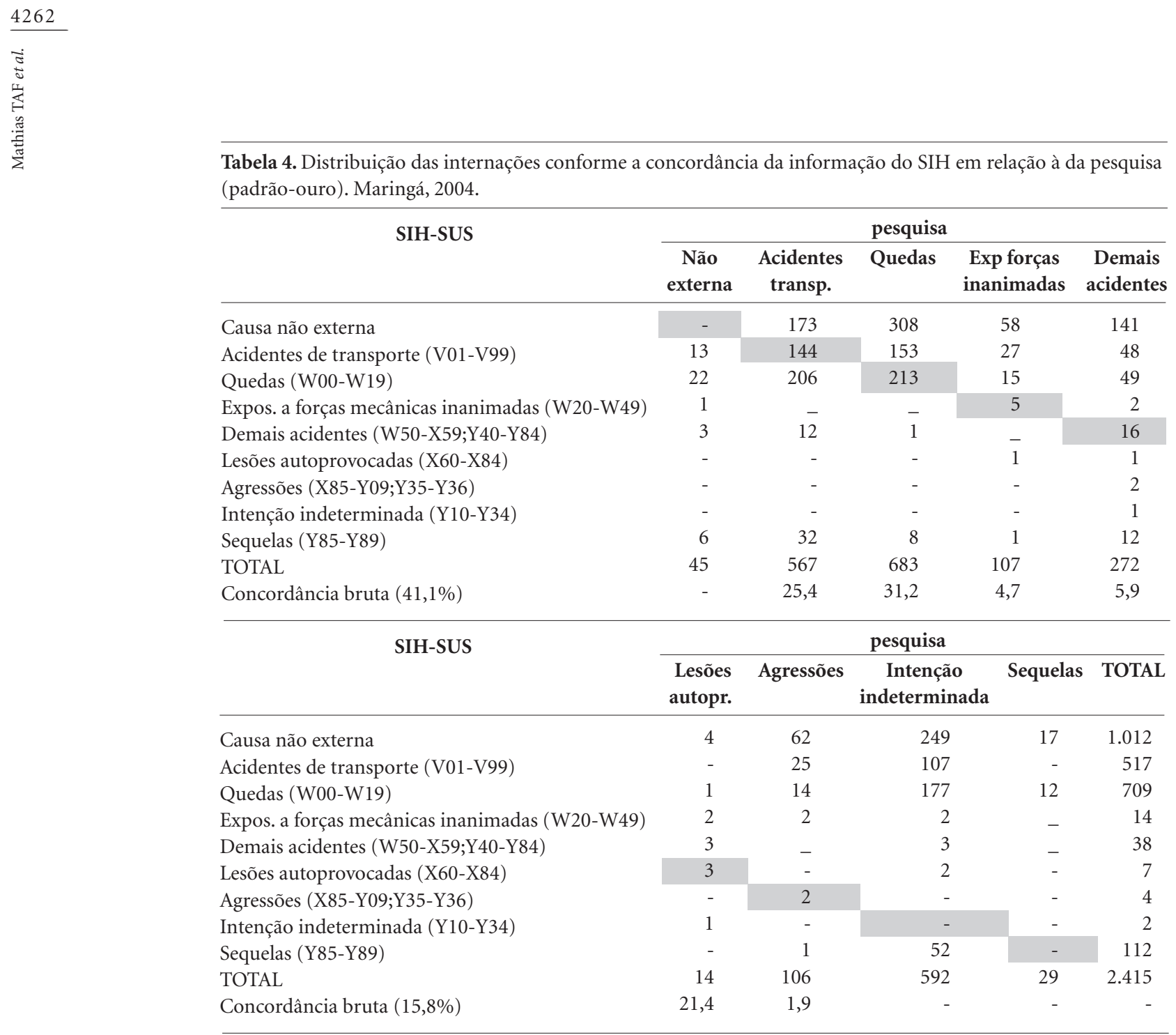

informação nos laudos e prontuários médicos, os trabalhadores dos hospitais codificam, preenchem e inserem os dados no sistema, da maior parte dos diagnósticos de internação, fato demonstrado pelo baixo percentual de causas externas de intenção indeterminada no SIH-SUS, encontrado tanto em Londrina $(1,9 \%)$ como em Maringá (0,1\%).

Em pesquisa realizada na Austrália, mais da metade dos codificadores clínicos entrevistados $(56,7 \%)$ afirmaram que a melhor qualidade dos dados sobre causas externas é encontrada nos registros das ambulâncias, ao contrário dos relatórios dos prontuários, que tinham informações insuficientes para uma codificação específica e detalhada das causas externas de internação. Melhorar a qualidade dos registros nos setores de emergência dos hospitais, providenciar formulários específicos para informações sobre causas externas foram as medidas mais citadas pelos entrevistados para facilitar e aprimorar a qualidade dos dados de causas externas de internações naquele país ${ }^{11,12}$.

Além do sub-registro para o total de causas externas, este estudo mostrou diferença na magnitude e na ordem de importância dos principais diagnósticos de internação quando o banco de dados da pesquisa foi comparado ao do SIHSUS. Enquanto no SIH-SUS predominaram as quedas, com 56,7\% e 50,6\% das internações em Londrina e Maringá, respectivamente, o banco pesquisa apontou em Londrina, os acidentes de transporte como as principais causas (28,3\%), com as quedas em segunda posição (24,3\%). Em Maringá, as duas primeiras causas de internação foram as mesmas apenas com inversão entre elas, pois o banco pesquisa mostrou que $28,8 \%$ eram quedas e $23,9 \%$ acidentes de transporte.

Observou-se que para as quedas houve boa concordância bruta apenas para Londrina 
(72,5\%), porém com confiabilidade apenas aceitável para esse município (kappa $=0,32$ ) e fraca para Maringá (kappa=0,07), concluindo-se que a maior parte da concordância bruta para o agrupamento das quedas ocorreu devido ao acaso, principalmente para Maringá. Em estudo que analisou causas externas de internação em hospitais no estado de Washington (EUA) foi encontrada boa concordância, tanto no banco oficial como no banco de dados corrigido, com as quedas em primeiro lugar e os acidentes de transporte em segundo na ordem de importância ${ }^{13}$.

A prevenção das quedas e dos acidentes de transporte, as duas principais causas externas de internação em ambos os municípios neste estudo, requer medidas específicas fundamentadas na especificidade e verdadeira magnitude desses eventos. Estudo que examinou a qualidade da codificação sobre causas externas em internações na Nova Zelândia observou que em somente 50\% das internações existia informação detalhada sobre o tipo de causa externa e que 57\% das internações por quedas foram codificadas em "W19", "quedas sem especificação"14. Da mesma forma, no presente estudo, grande parte das internações por quedas não foram detalhadas, ou seja, existia apenas a menção de "queda" no laudo médico ou no prontuário do paciente.

Análise das internações hospitalares sobre causas externas nos Estados Unidos observou que a qualidade dos dados é bastante variável entre os estados, sendo melhor naqueles onde existem regras de codificação bem estabelecidas e seu cumprimento é oficialmente controlado ${ }^{15}$. No Brasil, a informação sobre o diagnóstico de internação no banco do SIH-SUS varia de acordo com o tempo, o local e a natureza jurídica da instituição, e os funcionários ou as empresas contratadas nos setores administrativos dos hospitais não recebem treinamento para tratar o dado como necessário do ponto de vista de uma informação em saúde ${ }^{16}$. Em geral, não existe uma definição clara do tipo de formação necessária para os profissionais que atuam nos setores de arquivo médico e estatístico dos hospitais ${ }^{17}$. E para as internações financiadas pelo SUS a função administrativa e de reembolso dessas internações prevalece e privilegia as informações sobre os procedimentos realizados, ficando sem supervisão a qualidade das informações sobre os diagnósticos, principal e secundário de internação.

Destaca-se neste estudo que os registros hospitalares e outros oficiais sobre causas externas também apresentam fragilidades. Para a construção do banco de dados da pesquisa houve várias dificuldades devido à falta de informação da causa externa que gerou a lesão que motivou a internação, pois mesmo após a busca de informações em diferentes fontes e finalização da construção do banco de dados da pesquisa, cerca de $10 \%$ em Londrina e 25\% das internações em Maringá, permaneceram como causas externas de intenção indeterminada.

O presente estudo acrescentou um passo importante ao anterior ${ }^{7}$ na qualificação do banco de dados da pesquisa, que foi a busca aos prontuários em cada hospital de Londrina e Maringá. Este estudo acrescentou ainda informações sobre a confiabilidade das informações quando os agrupamentos de diagnósticos de causas externas são analisados em pares de registros revelando baixas concordâncias, porém com padrões diferenciados conforme os agrupamentos de causas e os municípios estudados.

Assim, fica evidente que os estudos sobre morbidade hospitalar no Brasil devem considerar tanto o fato de o SIH-SUS subestimar as causas externas nas internações por todas as causas, fato já descrito ${ }^{7}$, e as diferenças entre municípios e estados, como também que a distribuição dessas internações por agrupamentos de diagnósticos pode não refletir a real situação de morbidade hospitalar por acidentes e violências.

Mesmo com as restrições aqui relatadas em relação à qualidade dos dados sobre causas externas, somadas às restrições do caráter seletivo das estatísticas hospitalares, que permitem apenas o conhecimento dos casos mais graves que levam à internação e parcial, pois apenas parte do contingente dessas internações é conhecido ${ }^{18}$, o SIH-SUS deve ser valorizado. A análise dos dados deste banco tem permitido o conhecimento do perfil epidemiológico da morbidade hospitalar em geral e por lesões e causas externas em particular, sua magnitude, distribuição e gravidade, além de estimativas dos gastos públicos com essas internações ${ }^{19}$.

Este estudo evidenciou diferença dos diagnósticos de internação por causas externas, destacando o município de Londrina com dados de melhor qualidade. Portanto, considerando a variabilidade nas formas de codificar e consequente qualidade dos dados, é possível que estas diferenças sejam também encontradas entre outros municípios brasileiros. Em relação aos motivos da baixa cobertura e qualidade dos dados, outros estudos são necessários para essa avaliação, principalmente em Maringá, onde a maior concordância foi a aceitável, observada para as lesões autoprovocadas. Neste sentido, a qualidade das 
informações em saúde deve ser constantemente avaliada e aprimorada e os profissionais de saúde devem ser estimulados para a valorização dos sistemas de informação em saúde como fonte de informação para monitoramento e avaliação das ações, programas e políticas públicas.

Portanto, qualificar o SIH-SUS significa aperfeiçoar e potencializar a utilização de um sistema de informações em saúde universal e já consolidado para além da sua necessária função contábil, possibilitando dados confiáveis para análises epidemiológicas que contribuam para possíveis providências necessárias à viabilização e gerenciamento das atividades do setor público de saúde no Brasil, como conhecer e intervir na ocorrência de acidentes e violências em nosso país.

\section{Colaboradores}

TAF Mathias, RKT Souza, DFPP Soares e MPM Sapata participaram do desenvolvimento da pesquisa, da coleta e da análise de dados, e da redação do artigo. MFAI Tomimatsu e SM Andrade, participaram na concepção e desenvolvimento da pesquisa, coleta e análise de dados, redação do artigo. AS Frascarelli participou do desenvolvimento da pesquisa, da coleta de dados e da revisão final do artigo.

\section{Agradecimentos}

Ao Conselho Nacional de Desenvolvimento Científico e Tecnológico (CNPq) e ao Ministério da Saúde pelo apoio financeiro. 


\section{Referências}

1. World Health Organization (WHO). Violence, Injuries and Disability. Biennial 2006-2007 report. 2008. [acessado 2012 jan 28]. Disponivel em: http://bvsms.saude. gov.br/bvs/publicacoes/violence_injuries_disability. pdf

2. Brasil. Ministério da Saúde (MS). Departamento de Informática do Sistema Único de Saúde (Datasus). Mortalidade - Brasil. Óbitos por residência segundo capitulo da CID-9 e CID-10. [internet]. [acessado 2012 maio 3]. Disponível em: http://tabnet.datasus.gov.br/cgi/deftoh tm.exe?sim/cnv/obt10uf.def

3. Brasil. Ministério da Saúde (MS). Secretaria de Vigilância em Saúde. Departamento de Análise de Situação de Saúde. Viva: vigilância de violências e acidentes, 2006 e 2007. Brasília: MS; 2009. 154 p. [acessado 2012 jan 27]. Disponível em: http://bvsms.saude.gov.br/bvs/publicacoes/ viva_vigilancia_violencias_acidentes.pdf

4. Gawryszewski VP, da Silva MMA, Malta DC, Mascarenhas MDM, Costa VC, Matos SG, Moraes Neto OL, Monteiro RA, Carvalho CG, Magalhães ML. A proposta da rede de serviços sentinela como estratégia da vigilância de violências a acidentes. Cien Saude Colet 2007; 11(Supl.):1269-1278.

5. Bittencourt AS, Camacho LAB, Leal MC. O sistema de Informação Hospitalar e sua aplicação na saúde coletiva. Cad Saude Publica 2006; 22(1):19-30.

6. São Paulo. Secretaria de Estado da Saúde. Grupo Técnico de Prevenção de Acidentes e Violências. Centro de Vigilância Epidemiológica "Prof. Alexandre Vranjac". Coordenadoria de Controle de Doenças. Internações hospitalares por causas externas no Estado de São Paulo em 2005. Rev Saude Publica 2007; 41(1):163-166.

7. Tomimatsu MFA, Andrade SM, Soares DA, Mathias TAF Sapata MPM, Soares DFPP, Souza RKT. Qualidade da informação sobre causas externas no Sistema de Informações Hospitalares. Rev Saude Publica 2009, 43(3):413-420.

8. Cohen J. A coeficient of agreement for nominal scales. Educ Psychol Meas 1960; 20(1):37-46.

9. Abramson JH. WinPepi updated: computer programs for epidemiologists, and their teaching potential. Epidemiol Perspect Innov 2011; 8(1):1.

10. Landis JR, Koch GG. The measurement of observer agreement for categorical data. Biometrics 1977; 33(1):159-177.
11. McKenzie K, Harding LF, Walker SM, Harrison JE, Enraght-Moony EL, Waller GS. The quality of cause-of -injury data: where hospital records fall down. Aust $N$ Z J Public Health 2006; 30(6):509-513.

12. Mckenzie KM, Enraght-Moony E, Harding L, Walker S, Waller G, Chen L. Coding external causes of injuries: problems and solutions. Accid Anal Prev 2008; 40(2):714-718.

13. LeMier M, Cummings P, West TA. Accuracy of external cause of injury codes reported in Washington State hospital discharge records. Inj Prev 2001; 7(4):334-338.

14. Langley JD, Davie GS, Simpson JC. Quality of hospital discharge data for injury prevention. Inj Prev 2007; 13(1):42-44.

15. Coben JH, Steiner CA, Barrett M, Merrill CT, Adamson D. Completeness of cause of injury coding in healthcare administrative databases in the United States, 2001. Inj Prev 2006; 12(3):199-201.

16. Pepe VE. Sistema de informações hospitalares do Sistema Único de Saúde (SIH-SUS). In: Brasil. Ministério da Saúde (MS). A experiência brasileira em sistemas de informação em saúde. Vol. 2. Brasília: MS; 2009. p. 6586.

17. Schout D, Novaes HMD. Do registro ao indicador: gestão da produção da informação assistencial aos hospitais. Cien Saude Colet 2007; 12(4):935-944.

18. Lebrão MC, Mello Jorge MHP, Laurenti R. Morbidade hospitalar por lesões e envenenamentos. Rev Saude Publica 1997; 31(S4):26-37.

19. Mello Jorge MHP, Koizumi MS. Gastos governamentais do SUS com internações hospitalares por causas externas: análise no Estado de São Paulo, 2000. Rev Bras Epidemiol 2004; 7(2):228-238.

Artigo apresentado em 07/08/2013

Aprovado em 25/02/2014

Versão final apresentada em 09/03/2014 